OPEN ACCESS

Edited by:

Jean-Philippe Herbeuval,

UMR8601 Laboratoire de Chimie et Biochimie Pharmacologiques et

Toxicologiques, France

Reviewed by:

Mary K. Crow,

Hospital for Special Surgery, United States

Andras Perl,

Upstate Medical University, United States Gideon Schreiber

Weizmann Institute of Science, Israel

${ }^{*}$ Correspondence:

Alice Lepelley

alice.lepelley@institutimagine.org

Specialty section: This article was submitted to

Cytokines and Soluble

Mediators in Immunity,

a section of the journal

Frontiers in Immunology

Received: 23 June 2021 Accepted: 05 August 2021 Published: 26 August 2021

Citation:

Lepelley A, Wai T and Crow YJ (2021)

Mitochondrial Nucleic Acid as a Driver of Pathogenic Type I Interferon Induction in Mendelian Disease.

Front. Immunol. 12:729763. doi: 10.3389/fimmu.2021.729763

\section{Mitochondrial Nucleic Acid as a Driver of Pathogenic Type I Interferon Induction in Mendelian Disease}

\author{
Alice Lepelley ${ }^{1 *}$, Timothy Wai $^{2}$ and Yanick J. Crow ${ }^{1,3}$ \\ 1 Université de Paris, Imagine Institute, Laboratory of Neurogenetics and Neuroinflammation, Inserm UMR 1163, \\ Paris, France, ${ }^{2}$ Mitochondrial Biology Group, Institut Pasteur CNRS UMR 3691, Paris, France, ${ }^{3}$ Medical Research Council \\ Human Genetics Unit, Institute of Genetics and Cancer, University of Edinburgh, Edinburgh, United Kingdom
}

The immune response to viral infection involves the recognition of pathogen-derived nucleic acids by intracellular sensors, leading to type I interferon (IFN), and downstream IFNstimulated gene, induction. Ineffective discrimination of self from non-self nucleic acid can lead to autoinflammation, a phenomenon implicated in an increasing number of disease states, and well highlighted by the group of rare genetic disorders referred to as the type I interferonopathies. To understand the pathogenesis of these monogenic disorders, and polyfactorial diseases associated with pathogenic IFN upregulation, such as systemic lupus erythematosus and dermatomyositis, it is important to define the self-derived nucleic acid species responsible for such abnormal IFN induction. Recently, attention has focused on mitochondria as a novel source of immunogenic self nucleic acid. Best appreciated for their function in oxidative phosphorylation, metabolism and apoptosis, mitochondria are double membrane-bound organelles that represent vestigial bacteria in the cytosol of eukaryotic cells, containing their own DNA and RNA enclosed within the inner mitochondrial membrane. There is increasing recognition that a loss of mitochondrial integrity and compartmentalization can allow the release of mitochondrial nucleic acid into the cytosol, leading to IFN induction. Here, we provide recent insights into the potential of mitochondrialderived DNA and RNA to drive IFN production in Mendelian disease. Specifically, we summarize current understanding of how nucleic acids are detected as foreign when released into the cytosol, and then consider the findings implicating mitochondrial nucleic acid in type I interferonopathy disease states. Finally, we discuss the potential for IFN-driven pathology in primary mitochondrial disorders.

Keywords: type I interferonopathy, mitochondrial disease, type I interferon, autoinflammation, mitochondria, mtDNA, mtRNA, innate immunity

\section{INTRODUCTION}

Most cells are equipped with cytosolic sensors involved in the intracellular surveillance of pathogens, leading to the rapid induction of an antiviral IFN response (1). DNA is recognized by cyclic GMP-AMP synthase (cGAS), activating endoplasmic reticulum (ER)-resident Stimulator of interferon genes (STING) (2). STING then traffics to the Golgi, eventually inducing the 
transcription of IFN. Along similar lines, RNA species are recognized by RIG-I-like receptors (RLRs), RIG-I (retinoic acid-inducible gene I) and MDA5 (melanoma differentiationassociated protein 5), activating the adaptor protein mitochondrial antiviral-signaling protein (MAVS) on mitochondria, again leading to IFN induction (1).

The type I interferonopathies are rare genetic diseases characterized by chronic upregulation of type I IFN signaling (3). Strikingly, the majority of type I interferonopathy-related disease genes identified to date encode molecules playing a role in nucleic acid processing or sensing, highlighting the importance of active mechanisms to prevent antiviral responses triggered by self nucleic acids, and the challenge of self/non-self discrimination (4). Indeed, aberrant sensing of self nucleic acids has been increasingly implicated in a diversity of pathologies including autoimmunity, genome instability syndromes, cancer, neurodegeneration and senescence (4).

To better understand pathogenesis, it is important to determine the source of the self nucleic acids detected by innate (antiviral) sensors. Recent studies have established that genomic DNA represents such an agonist when abnormally exposed to cGAS (2,5-7). Interestingly, in some type I interferonopathies, and in senescence, DNA and RNA derived from endogenous retroelement expression may also represent 'self'-derived nucleic acid capable of triggering IFN signaling (812). Notably, beyond the nucleus, mitochondria constitute the other intracellular source of self nucleic acids, possessing their own DNA (mtDNA) and RNA (mtRNA) enclosed by the mitochondrial membranes (13). The mtDNA encodes 13 respiratory chain proteins, 22 tRNAs and 2 rRNAs, with the remaining $\sim 1,300$ mitochondrial proteins imported after translation from the nuclear genome (13). Since mitochondria constitute the cytosolic remnants of the endosymbiosis of proteobacteria within eukaryotic cells (14), mtDNA and mtRNA demonstrate immunostimulatory characteristics of pathogens, with the potential to be misinterpreted as foreign. Thus, each cell contains hundreds to thousands of copies of circular double stranded (ds) mtDNA molecules, which are hypomethylated, devoid of histones, exposed to reactive oxygen species (ROS) and poorly repaired. Furthermore, bidirectional transcription generates long dsRNA and uncapped mRNAs, and mtDNA transcription and replication give rise to single stranded DNA, RNA-DNA hybrids and Gquadruplexes $(13,15,16)$, all of which have immunostimulatory capacity. Indeed, there is increasing recognition of the potential of mitochondrial-derived nucleic acids (mtNA) to act as agonists of the IFN signaling machinery, possibly contributing to complex autoinflammatory diseases such as systemic lupus erythematosus (SLE) (17), as well as neurodegeneration $(18,19)$ and cancer $(20,21)$.

In this review we focus on mtNA cytosolic sensing leading to IFN induction. However, we note that mtDNA and other mitochondrial molecules [cardiolipins, formyl peptides, mitochondrial ROS (mROS)] can also trigger other innate sensing pathways. For example, these molecules can engage the inflammasome, resulting in interleukin $1 \beta$ (IL1 $\beta$ )-mediated inflammation (22-25) and endosomal Toll-like receptor 9 (TLR9) activation, leading to IFN and NF- $\kappa \mathrm{B}$-dependent inflammatory cytokine induction $(26,27)$. These pathways, also implicated in inflammatory diseases, are specific to discrete cell types and have been extensively reviewed elsewhere $(15,24,25,28,29)$. mtDNA can also be released into the extracellular space, acting as a plasmatic marker and driver of systemic inflammation in autoimmunity, traumatic injury, lung inflammation and cardiovascular disease $(30,31)$.

\section{MITOCHONDRIAL NUCLEIC ACID IS INTERFERONOGENIC IN THE CYTOSOL}

mtNA remains 'immunologically inert' when retained inside the two nested compartments delimited by the mitochondrial membranes: the matrix enclosed in the inner mitochondrial membrane (IMM), and the inner membrane space (IMS) between the IMM and the outer mitochondrial membrane (OMM) (25) (Figure 1). Thus, an increasing number of reports indicate that loss of mitochondrial integrity and compartmentalization, as a result of mitochondrial stress, can allow the release of mtNA, and subsequent interaction with cytosolic receptors leading to IFN induction $(25,28)$.

Seminal studies first described IFN induction due to mtDNA release upon abortive apoptosis in $2014(32,33)$, the process of which was detailed in real-time by high-resolution imaging in $2018(34,35)$. Upon mitochondrial apoptosis triggered by the activation of BAX (Bcl-2 associated-X protein) and BAK (BCL-2 homologous antagonist/killer), BAX/BAK pores are formed in the OMM, releasing proapoptotic factors from the IMS, and leading to activation of caspases 9,3 and 7 and apoptotic cell death (36). However, when BAX/BAK activation is induced together with caspase inhibition, mtDNA complexed to TFAM into nucleoids is released into the cytosol, sensed by cGASSTING, and IFN induced $(32,33)$, suggesting a role for programmed cell death completion in preventing inflammatory mtNA sensing. Despite these insights, the question of how mtDNA might first cross the IMM remained. A contribution of the mitochondrial permeability transition pore (mPTP), an IMM channel that allows for non-selective diffusion of low molecular weight solutes and water $(<1.5 \mathrm{kDa})(37)$, as well as mROS-dependent destabilization of the IMM, have been suggested to facilitate such egress $(23,30,38,39)$. Recent work has also highlighted IMM herniation, through BAX/BAK macropores, followed by IMM permeabilization independent of mPTP opening $(34,35)$ (Figure 1). However, these mechanisms of IMM crossing are difficult to reconcile with the size of mtDNA nucleoids (40).

Remarkably, an increasing number of situations associated with mitochondrial stress have been linked to the release and sensing of mtNA, mostly mtDNA through cGAS-STING, and the induction of IFN (28). These include environmental insults, oxidative stress, 'suboptimal' mitochondrial function, mitochondrial dysfunction due to mutations (mitochondrial disease detailed below), 


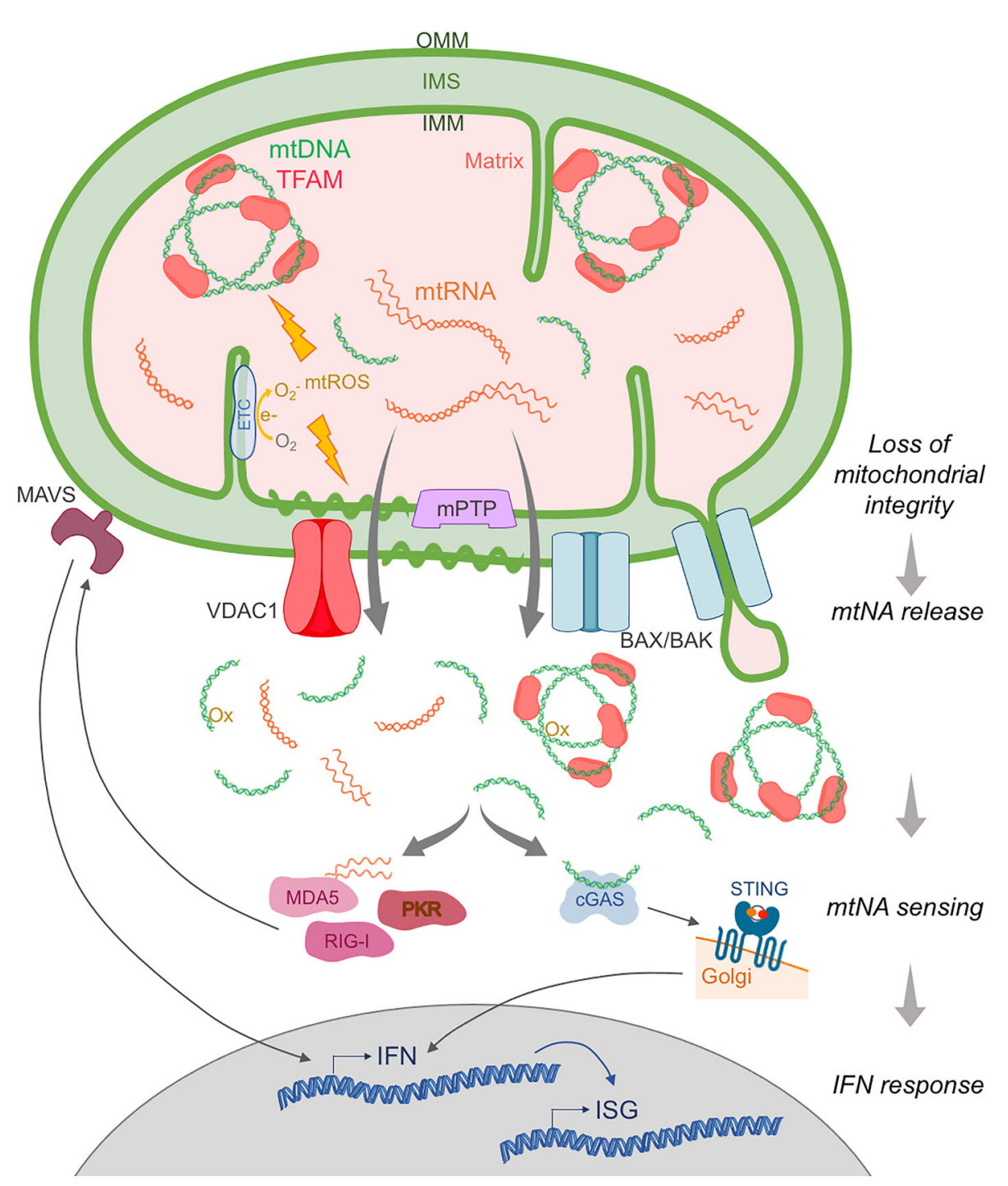

FIGURE 1 | Main pathways of mitochondrial nucleic acid release and sensing. Upon extrinsic or intrinsic insult, the mitochondrial membrane integrity is compromised and mtDNA and mtRNA, normally contained within the double membrane, can be released into the cytosol. Release of mtDNA packaged into nucleoids by TFAM is mediated by outer mitochondrial membrane (OMM) perforation by BAX/BAK macropores, while mtDNA fragments devoid of TFAM are thought to egress through VDAC1 pores. Inner mitochondrial membrane (IMM) permeabilization to mtNA can involve herniation into BAX/BAK pores, destabilization due to oxidative stress (e.g. mitochondrial ROS (mtROS) generated from electrons (e_) leaking from the electron transport chain (ETC)), and/or opening of the mitochondrial permeability transition pore (mPTP). In the cytosol, mtDNA, oxidized (Ox) mtDNA and mtRNA are detected as foreign by innate cytosolic sensors of immunostimulatory DNA (e.g. cGAS), and RNA (e.g. RIG-I, MDA5, PKR). These receptors then activate the adaptor molecules STING and MAVS, respectively, leading to the induction of IFN and subsequent IFN-stimulated gene (ISG) expression. IMS, inner membrane space.

proteotoxic stress, and infection $(24,25,30)$. Such stress, typically evidenced by impaired oxidative phosphorylation and ATP production, metabolic imbalance, loss of mitochondrial potential and mROS induction, results in a loss of mitochondrial integrity and release of mitochondrial components. Notably, this phenomenon can be considered 'physiological' when induced by pathogens, promoting an antiviral state (41-45). Cytosolic mtDNA then constitutes a second messenger, initiating the antiviral response. Along similar lines, upon genotoxic stress, mtNA release is sensed as a sign of genomic instability and can activate
DNA repair pathways $(20,46,47)$. Interestingly, in the context of SLE, mitochondrial oxidative stress enhances the interferonogenic potential of mtDNA itself by oxidation (17, 48, 49) (Figure 1). Indeed, mitochondrial hyperpolarization causes slippage of electrons onto molecular oxygen, which is reversible by treatment with the antioxidant $\mathrm{N}$-acetylcysteine in vitro and in vivo; also demonstrating therapeutic efficacy in patients with $\operatorname{SLE}(50,51)$.

Studies of mtNA release upon different mitochondrial stresses have both reinforced the role of BAX/BAK macropores $(21,43$, $46,52,53)$ and mPTP $(17,54)$, and elucidated further relevant 
mechanisms and the type of mtDNA species egressing. As an example, upon loss of mitochondrial endonuclease EndoG, oxidative stress triggers voltage-dependent anion-selective channel 1 (VDAC1) oligomerization and the formation of pores in the OMM, with subsequent release into the cytosol of mtDNA fragments, rather than TFAM-bound nucleoids, a situation relevant to SLE (17), viral infection (55) and altered mitochondrial proteostasis (56) (Figure 1). In some instances, a combination of VDAC1 pores in the OMM, and MPTP for IMM permeabilization, facilitates complete mitochondrial envelope opening $(17,19)$. In the case of infection, viroporins and other microbial proteins have been proposed to perforate mitochondrial membranes $(43,57)$. Host inflammasome effector Gasdermin D can also permeabilize mitochondria (58), and the RLR adaptor MAVS has been described as a mitochondrial membrane remodeler $(43,59,60)$, although the release of mtNA remains to be observed in this situation.

Analogous to the processes involving mtDNA described above, a few studies have reported mtRNA relocalization leading to innate immune stimulation. Upon loss of p53, mtRNA is sensed by cytosolic MDA5 and RIG-I (61), possibly related to the opening of the MPTP (62). In a model of Huntington's disease, mtRNA accumulated in the cytosol, activating the RNA sensor protein kinase $\mathrm{R}$ (PKR) and subsequent IFN-stimulated gene (ISG) expression (63). Interestingly, mtDNA ds breaks can lead to mtRNA sensing by RIG-I in the cytosol (46). Additionally, mtRNA sensing might be relevant in the context of TLR7dependent IFN induction upon ablation of the mitophagy actor IRGM1 in mouse macrophages (TLR7 being an RNA sensor) (64), and in the RNA sensing/MAVS-dependent mtDNA cytosolic leakage upon influenza virus M2 viroporin expression (43).

Although speculative, loss of membrane integrity might also allow the entry of nucleic acid sensors into mitochondria. Indeed, mitochondrial membranes contain complexes involved in the import of nuclear-encoded mitochondrial proteins (65), enabling, for example, aberrant entry of mutated TDP-43 in the context of amyotrophic lateral sclerosis (ALS) (19). This phenomenon could explain the observation of a basal interaction between PKR and mitochondrial dsRNA (66). Interestingly, although cGAS recruitment to IMM hernias, for mtDNA sensing, is not observed during abortive apoptosis (35), cGAS contains a cryptic mitochondrial targeting sequence, and truncated cGAS can translocate to mitochondria and become activated (67).

Mitophagy constitutes the selective degradation of damaged mitochondria by autophagy, participating in mitochondrial quality control (25). A priori, removal of dysfunctional mitochondria leaking mtNA may act as a safeguard against detrimental IFN induction. Thus, although not directly implicated in mtNA retention, mitophagy activation could limit mitochondrial immunogenicity and pathogenicity (68). As such, autophagy and mitophagy have been shown to dampen the innate immune response induced by mtNA leakage and sensing $(17,21,23,27$, $69,70)$, and, even, to be triggered by pathogens themselves $(71,72)$. Consistently, defective mitophagy can enhance sensing $(18,73)$ [reviewed in $(24,25,74)$ ]. Importantly, such 'mitophagic maintenance' has been suggested to have pathogenic relevance in autoimmune diseases [e.g. Sjögren's syndrome (64) and SLE (75)], and Parkinson's disease (18).

Summarizing, our understanding of how mitochondrial stress and damage leads to mitochondrial rupture and IFN-inducing mtNA release has recently broadened. However, these data have been mostly derived from in vitro studies, mouse models, or through biomarker correlations (28-30).

\section{IMMUNOSTIMULATORY MITOCHONDRIAL NUCLEIC ACID IN TYPE I INTERFERONOPATHIES}

Providing strong evidence of the potential of mtNA to induce pathological IFN induction in humans, inappropriate sensing of mtNA has recently been demonstrated in Mendelian diseases due to mutations in PNPT1, NGLY1 and ATAD3A $(73,76,77)$ (Figure 2). These observations highlight mechanisms involved in mitochondrial homeostasis directly relevant to the avoidance of aberrant sensing of mtNA in human health.

Dhir et al. described loss of the mtRNA exoribonuclease PNPT1 to result in an accumulation and cytosolic leakage of dsRNA derived from bidirectional mtDNA transcription, triggering IFN through a BAX/BAK-dependent mechanism (76). Consistent with the type I interferonopathy disease spectrum, patients carrying hypomorphic mutations in PNPT1 display enhanced IFN signaling in blood (and, in some cases, intracerebral calcification, a well-known clinical feature of IFN activation) $(76,78)$. Mutations in NGLY1, encoding conserved deglycosylation enzyme NGLY1, cause a severe neurodevelopmental phenotype $(79,80)$. In a mouse model, Yang et al. showed that loss of NGLY1 also results in chronic activation of cytosolic nucleic acid sensing pathways, likely induced by a combination of mtRNA and mtDNA (73). Here, mitochondrial quality control may be the broad link between NGLY1 and mtNA homeostasis, involving mitophagy and/or proteasome function. Relating to the clinical phenotype, an apparent resistance to viral infection was noted, and increased ISG expression recorded in patient-derived cell lines, although the contribution of IFN to the observed neuropathology remains to be defined (73).

Very recently, we directly implicated, for the first time, mtDNA sensing in IFN induction in a Mendelian disease context (77). Specifically, we identified two patients demonstrating chronically enhanced IFN signaling in blood and features of systemic sclerosis, a rare autoimmune disorder where IFN signaling and mtDNA have been suggested to play a role in pathogenesis (81-83). Surprisingly, these patients carried dominant negative heterozygous mutations in $A T A D 3 A$, encoding the mitochondrial AAA ATPase protein ATAD3A, previously described to cause mitochondrial disease with neurological features $(84,85)$. Importantly, we also observed enhanced IFN signaling in patients with a predominant neurological clinical phenotype, suggesting a consistent link between ATAD3A mutations and IFN signaling. We demonstrated cytosolic leakage of mtDNA, and cGAS-STING-dependent IFN induction (Figure 2). Although VDAC1 oligomers seemed to be relevant, the mechanisms of mtDNA cytosolic relocalization will require further study, and a direct role for ATAD3A cannot be excluded. Indeed, ATAD3A has 


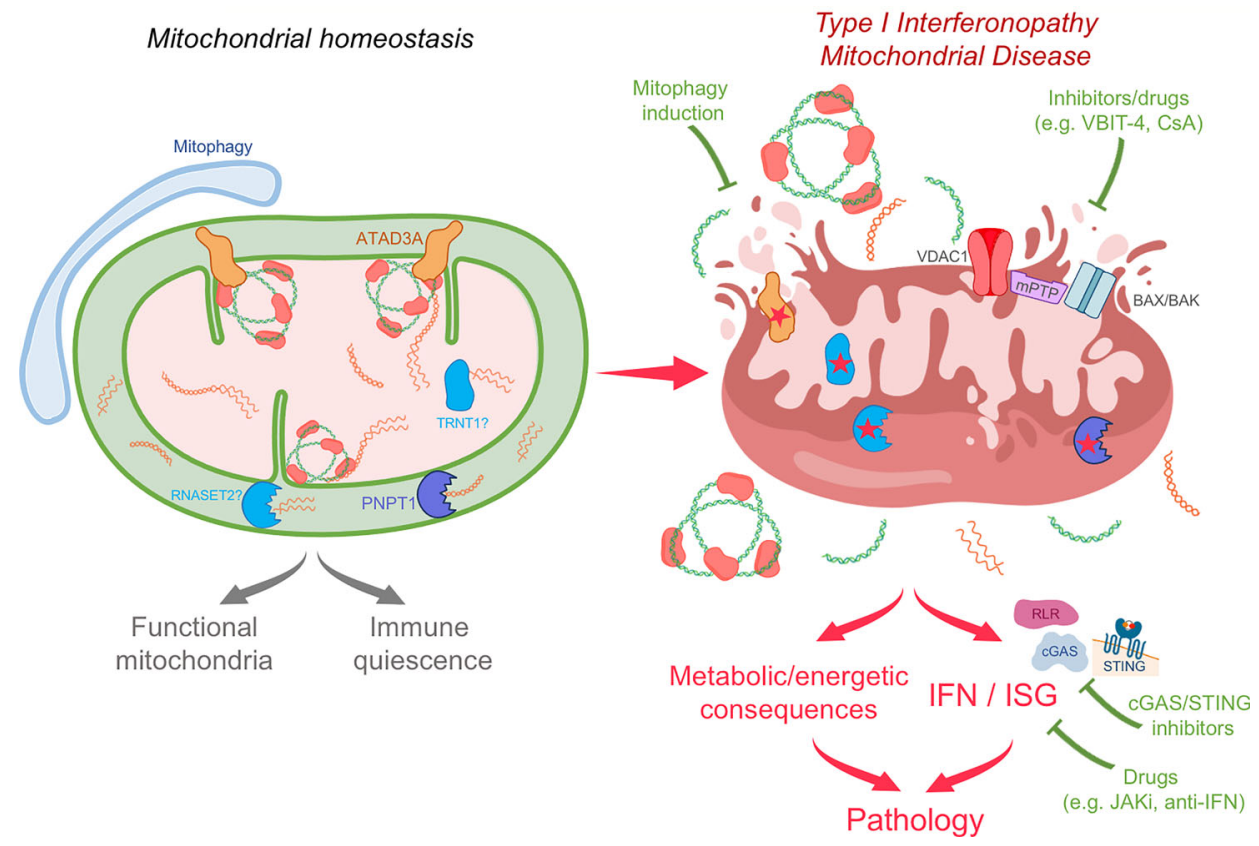

FIGURE 2 | Potentially common pathogenic mechanisms in type I interferonopathy and mitochondrial disease and therapeutic perspectives. Due to the immunostimulatory potential of mtNA, active processes are required to ensure mitochondrial homeostasis and immunological quiescence. These include degradation of mtdsRNA by PNPT1, maintenance of mtDNA and mitochondrial structure by molecules such as ATAD3A, and metabolism of mtRNA by proteins such as RNASET2 and TRNT1. More general mitochondrial quality control mechanisms are involved as well, including mitophagy and mitochondrial proteases. Defects in these processes may result in both mitochondrial disease and a type I interferonopathy state. Indeed, loss of function of PNPT1 and ATAD3A, and possibly of RNASET2 and TRNT1, can lead to mitochondrial stress and mtNA cytosolic leakage and sensing. In the type I interferonopathies linked to mutations in PNPT1 and ATAD3A, in addition to mitochondrial dysfunction, there is chronic induction of IFN and ISGs. This might also be a feature of other mitochondrial diseases, which may have gone undetected due to lack of relevant investigations. Identifying immunostimulatory mtNA and IFN induction as pathogenic mechanisms opens new therapeutic perspectives, including by inhibition of type I IFN signaling (JAK inhibitors (JAKi), anti-IFN system antibodies), mtNA sensing (cGAS/STING inhibitors), mitochondrial membrane opening [VBIT-4, cyclosporin A (CsA)], or by mitophagy induction.

been implicated in multiple mitochondrial processes, including mtDNA maintenance, mitochondrial ultrastructural organization, mitochondria-ER junction stabilization and cholesterol biosynthesis (86-90).

Interestingly, hypomorphic mutations in RNASET2 and TRNT1 may also be associated with perturbed mtNA homeostasis leading to enhanced IFN signaling $(91,92)$. Mutations in RNASET2 cause a phenotype mimicking congenital viral infection, reminiscent of some type I interferonopathies $(78,93)$, and RNASET2 has been suggested to play a role in mitochondrial ribosomal RNA degradation in the IMS (94). Further, IFN pathway induction has been observed in some patients with mutations in TRNT1 (91), and TRNT1 is required for tRNA aminoacylation of mitochondrial and cytosolic tRNA, with protein dysfunction leading to defective mitochondrial translation (95). Of interest, a Mendelian metabolic disease due to deficiency in mevalonate kinase, involved in the biosynthesis of cholesterol and isoprenoids, may also involve mitochondrial damage, mtDNA release and sensing (96). However, to date, only inflammasome pathway activation, leading to IL1 $\beta$ induction, has been implicated mechanistically (97). Since anti-IL1 $\beta$ signaling treatments are only partially effective in this context $(98,99)$, one might speculate that enhanced IFN signaling may be contributive to the phenotype (100-102). We also note that in iPSC-derived motor neurons from patients carrying TDP-43 mutations associated with ALS, mtDNA release and sensing lead to IFN induction, although the relevance of IFN signaling to ALS remains unclear (19).

Interestingly, mtRNA relocalization and sensing have been only infrequently implicated in the numerous studies reporting in vitro mitochondrial stress leading to IFN induction $(46,61,63,64)$. However, given that mtDNA depletion, used in vitro to prove the implication of mtDNA, also results in mtRNA depletion, mtRNA may have a currently unappreciated role in this context (even when demonstrating DNA-dependent sensing). Further, since the majority of dsRNA detected in the cytosol is of mitochondrial origin (28), and PKR binds mtRNA at steady state (66), constitutive leakage of mtRNA may prevent the recording of acute mtRNA sensing. Indeed, mtRNA may be more 'mobile' than mtDNA, since it is untethered to the mitochondrial membrane (unlike mtDNA nucleoids organized around TFAM) (13). Alternatively, it may be that mtRNA abundance and containment are tightly regulated, with redundant mitochondrial and cytosolic nucleases preventing their accumulation $(103,104)$, and/or that mtRNA cytosolic leakage and sensing are more harmful to cells in vitro, leading to toxic translational arrest through activation of the PKR pathway $(66,105)$. 
The above cases illustrate the potential of the study of type I IFNrelated Mendelian disease to define novel cellular functions, revealed by hypomorphic or gain-of-function mutations, providing insights into poorly understood mechanisms of mtNA retention.

\section{AN OVERLAP BETWEEN TYPE I INTERFERONOPATHY AND MITOCHONDRIAL DISEASE?}

Mutations in more than 350 nuclear or mtDNA-encoded genes are known to result in mitochondrial disease, involving diverse tissues and responsible for heterogenous clinical phenotypes $(106,107)$. Clinical characterization is lengthy and difficult, and, where a genetic diagnosis is unavailable, relies on the identification of metabolic changes, neuropathological manifestations and mitochondrial dysfunction in muscle biopsy $(106,108,109)$. While the contribution of defective oxidative phosphorylation and bioenergetic and metabolic stress is clear, the findings summarized above suggest that mtNA sensing driving IFN signaling may also be relevant to mitochondrial disease pathology. Thus, disease caused by mutations in PNPT1 and $A T A D 3 A$ are considered as bona fide primary mitochondrial disorders, and might now also be included in the type I interferonopathy grouping $(76,77,106)$. Mitochondrial disease is typically accompanied by various types of mitochondrial dysfunction and/or due to specific defects in mtNA metabolism $(106,108)$, with the potential to cause mtNA release and sensing. Interestingly, it has been suggested that enhanced IFN signaling, linked to mtDNA cytosolic release, can occur in mitochondrial syndromes due to single large-scale mtDNA deletions, associated with clinical features overlapping with those seen in certain type I interferonopathies (such as basal ganglia calcification and skin lesions) (110). Additionally, some mitochondrial disease mouse models manifest exacerbated IFN signaling associated with engagement of cytosolic mtDNA sensing, e.g. upon loss of the mitochondrial proteases CLPP and YMEL1 $(56,111)$. Similarly, multisystemic dysfunction caused by mtDNA mutation accumulation in the proofreading-deficient POLG mutator mouse can be rescued by ablation of cGAS-STING activity or IFN signaling (112). Whether maladaptive inflammation is observed in the corresponding human mitochondrial diseases has not been explored.

It is important to emphasize that the evaluation of IFN signaling is still not routine in medical practice $(113,114)$, even for inflammatory diseases. Thus, even if autoinflammation is not typically reported in mitochondrial disease (115-117), we suggest that increased IFN signaling may be more broadly associated with mitochondrial dysfunction than is currently appreciated, potentially contributing to the clinical phenotype beyond bioenergetic or metabolic defects. Indeed, enhanced interferon signaling related to ATAD3A dysfunction was only recognized six years after gene mutations were initially described $(77,84,85)$. Further indication of a possible relationship between mitochondrial disease and the type I interferonopathies comes from shared clinical features, such as intracranial calcification being an established sign in both settings $(3,118)$. Similarly, bilateral striatal necrosis is recurrent in mitochondrial disease, and consistently described in the context of mutations in both PNPT1 (78) and the type I interferonopathy due to ADAR1 loss-of-function (119). Likewise, dystonia, peripheral neuropathy, hypertrophic cardiomyopathy and isolated spastic paraparesis, recorded in patients with mutations in $A T A D 3 A(77,84,85)$, are features of interferonrelated disease $(120,121)$.

\section{PERSPECTIVES}

The power of studying Mendelian diseases lies in the deconvolution of complex processes relevant to human health. Thus, if further validated, an overlap between type I IFN-related and mitochondrial diseases would, in combination, facilitate our understanding of the safeguards in place to prevent inappropriate mtNA sensing leading to harmful IFN induction. In vitro screening approaches using knock down strategies are hampered by the potential induction of cellular toxicity, and do not necessarily afford the mechanistic insights that studying gain-offunction and hypomorphic mutations can provide. Indeed, the diversity of processes described so far as contributing to mtNA leakage and sensing upon mitochondrial stress, suggests that the immunological quiescence of mtNA is achieved through currently incompletely understood, and difficult to predict, active processes.

Clearly, the extent and significance of an overlap between mitochondrial dysfunction and type I IFN induction in human disease needs to be defined, perhaps foremost by the systematic screening of IFN signaling status in the blood and cerebrospinal fluid of mitochondrial disease patients. Such studies could have important clinical implications, both from a diagnostic and therapeutic perspective. Thus, therapies targeting IFN signaling, and showing clinical benefits in type I interferonopathies, are available (JAK inhibition) $(122,123)$, and others are in development [e.g. anti-IFN (receptors) antibodies and STING inhibitors] (28, 124, 125) (Figure 2). These could provide a new therapeutic angle for mitochondrial disorders, most lacking real treatment options (126). Proving the contribution of pathogenic IFN signaling to disease will require the observation of clinical improvement with such IFNtargeted therapies. Therapeutic approaches could also target broader processes beyond blocking IFN signaling in diseases implicating mtNA sensing, e.g. through the removal of ruptured mitochondria by inducing mitophagy $(17,74,127,128)$ (Figure 2). In this regard, two patients with mutations in $A T A D 3 A$ have been treated for inflammatory features by rapamycin (77), used as an immunosuppressant (129), but which, one could speculate, may act as a mitophagy inducer in this case. Indeed, rapamycin has shown benefit in a few patients with a mitochondrial encephalopathy (130), and a clinical trial of rapamycin is planned for the mitochondrial disorder Leigh syndrome (126).

Taking account of the potential sensing of escaped mtNA in mitochondrial disease might shed light on pathogenesis, and explain poorly understood features of these diseases such as variable clinical penetrance, specificity of tissue involvement only partially correlated to bioenergetic demands, and exacerbation of mitochondrial disease after infection or metabolic challenge 
$(106,109)$. Conversely, mitochondrial damage due to mutations in mitochondrial genes as a cause of type I interferonopathies lacking a genetic cause is also worthy of closer consideration.

\section{AUTHOR CONTRIBUTIONS}

AL wrote the first draft of the manuscript and designed figures. TW and YC provided valuable comments and edited the manuscript. All authors contributed to the article and approved the submitted version.

\section{FUNDING}

AL is supported by funding from the European Union's Horizon 2020 research and innovation program under the Marie

\section{REFERENCES}

1. Ablasser A, Hur S. Regulation of cGAS- and RLR-Mediated Immunity to Nucleic Acids. Nat Immunol (2019) 21(1):17-29. doi: 10.1038/s41590-019-0556-1

2. Decout A, Katz JD, Venkatraman S, Ablasser A. The cGAS-STING Pathway as a Therapeutic Target in Inflammatory Diseases. Nat Rev Immunol (2021) 8:1-22. doi: 10.1038/s41577-021-00524-Z

3. Rodero MP, Crow YJ. Type I Interferon-Mediated Monogenic Autoinflammation: The Type I Interferonopathies, a Conceptual Overview. J Exp Med (2016) 213(12):2527-38. doi: 10.1084/jem.20161596

4. Uggenti C, Lepelley A, Crow YJ. Self-Awareness: Nucleic Acid-Driven Inflammation and the Type I Interferonopathies. Annu Rev Immunol (2019) 37:247-67. doi: 10.1146/annurev-immunol-042718-041257

5. Mackenzie KJ, Carroll P, Martin CA, Murina O, Fluteau A, Simpson DJ, et al. cGAS Surveillance of Micronuclei Links Genome Instability to Innate Immunity. Nature (2017) 548(7668):461-5. doi: 10.1038/nature23449

6. Guey B, Wischnewski M, Decout A, Makasheva K, Kaynak M, Sakar MS, et al. BAF Restricts cGAS on Nuclear DNA to Prevent Innate Immune Activation. Science (2020) 369(6505):823-8. doi: 10.1126/science.aaw6421

7. Uggenti C, Lepelley A, Depp M, Badrock AP, Rodero MP, El-Daher MT, et al. cGAS-Mediated Induction of Type I Interferon Due to Inborn Errors of Histone pre-mRNA Processing. Nat Genet (2020) 52(12):1364-72. doi: 10.1038/s41588-020-00737-3

8. Stetson DB, Ko JS, Heidmann T, Medzhitov R. Trex1 Prevents Cell-Intrinsic Initiation of Autoimmunity. Cell (2008) 134(4):587-98. doi: 10.1016/ j.cell.2008.06.032

9. Thomas CA, Tejwani L, Trujillo CA, Negraes PD, Herai RH, Mesci P, et al. Modeling of TREX1-Dependent Autoimmune Disease Using Human Stem Cells Highlights L1 Accumulation as a Source of Neuroinflammation. Cell Stem Cell (2017) 21(3):319-31.e8. doi: 10.1016/j.stem.2017.07.009

10. De Cecco M, Ito T, Petrashen AP, Elias AE, Skvir NJ, Criscione SW, et al. L1 Drives IFN in Senescent Cells and Promotes Age-Associated Inflammation. Nature (2019) 566(7742):73-8. doi: 10.1038/s41586-018-0784-9

11. Rice GI, Meyzer C, Bouazza N, Hully M, Boddaert N, Semeraro M, et al. Reverse-Transcriptase Inhibitors in the Aicardi-Goutieres Syndrome. N Engl J Med (2018) 379(23):2275-7. doi: 10.1056/NEJMc1810983

12. Chung H, Calis JJA, Wu X, Sun T, Yu Y, Sarbanes SL, et al. Human ADAR1 Prevents Endogenous RNA From Triggering Translational Shutdown. Cell (2018) 172(4):811-24.e14. doi: 10.1016/j.cell.2017.12.038

13. Fu Y, Tigano M, Sfeir A. Safeguarding Mitochondrial Genomes in Higher Eukaryotes. Nat Struct Mol Biol (2020) 27(8):687-95. doi: 10.1038/s41594020-0474-9

14. Youle RJ. Mitochondria-Striking a Balance Between Host and Endosymbiont. Science (2019) 365(6454):eaaw9855. doi: 10.1126/ science.aaw 9855
Skłodowska-Curie grant agreement No. 892311. YC acknowledges that work relating to this manuscript has received funding from the European Research Council (ERC) under the European Union's Horizon 2020 research and innovation programme (grant agreement No 786142) and State funding from the Agence Nationale de la Recherche under "Investissements d'avenir" program (ANR-10-IAHU-01). TW acknowledges funding from the ERC (grant agreement No. 714472)

\section{ACKNOWLEDGMENTS}

Due to space constraints, we apologize to authors whose work could not be mentioned in detail in this mini review. We thank Ashish Dhir, Marie-Louise Frémond and Isabelle Melki for helpful discussions.

15. West AP, Shadel GS. Mitochondrial DNA in Innate Immune Responses and Inflammatory Pathology. Nat Rev Immunol (2017) 17(6):363-75. doi 10.1038/nri.2017.21

16. Falabella M, Fernandez RJ, Johnson FB, Kaufman BA. Potential Roles for GQuadruplexes in Mitochondria. Curr Med Chem (2019) 26(16):2918-32. doi: $10.2174 / 0929867325666180228165527$

17. Kim J, Gupta R, Blanco LP, Yang S, Shteinfer-Kuzmine A, Wang K, et al. VDAC Oligomers Form Mitochondrial Pores to Release mtDNA Fragments and Promote Lupus-Like Disease. Science (2019) 366(6472):1531-6. doi: 10.1126/science.aav4011

18. Sliter DA, Martinez J, Hao L, Chen X, Sun N, Fischer TD, et al. Parkin and PINK1 Mitigate STING-Induced Inflammation. Nature (2018) 561 (7722):258-62. doi: 10.1038/s41586-018-0448-9

19. Yu CH, Davidson S, Harapas CR, Hilton JB, Mlodzianoski MJ, Laohamonthonkul P, et al. TDP-43 Triggers Mitochondrial DNA Release via mPTP to Activate cGAS/STING in ALS. Cell (2020) 183(3):636-49.e18. doi: 10.1016/j.cell.2020.09.020

20. Wu Z, Oeck S, West AP, Mangalhara KC, Sainz AG, Newman LE, et al Mitochondrial DNA Stress Signalling Protects the Nuclear Genome. Nat Metab (2019) 1(12):1209-18. doi: 10.1038/s42255-019-0150-8

21. Yamazaki T, Kirchmair A, Sato A, Buque A, Rybstein M, Petroni G, et al. Mitochondrial DNA Drives Abscopal Responses to Radiation That are Inhibited by Autophagy. Nat Immunol (2020) 21(10):1160-71. doi: 10.1038/s41590-020-0751-0

22. Zhou R, Yazdi AS, Menu P, Tschopp J. A Role for Mitochondria in NLRP3 Inflammasome Activation. Nature (2011) 469(7329):221-5. doi: 10.1038/ nature 09663

23. Nakahira K, Haspel JA, Rathinam VA, Lee SJ, Dolinay T, Lam HC, et al. Autophagy Proteins Regulate Innate Immune Responses by Inhibiting the Release of Mitochondrial DNA Mediated by the NALP3 Inflammasome. Nat Immunol (2011) 12(3):222-30. doi: 10.1038/ni.1980

24. Song Y, Zhou Y, Zhou X. The Role of Mitophagy in Innate Immune Responses Triggered by Mitochondrial Stress. Cell Commun Signal (2020) 18(1):186. doi: 10.1186/s12964-020-00659-x

25. Moehlman AT, Youle RJ. Mitochondrial Quality Control and Restraining Innate Immunity. Annu Rev Cell Dev Biol (2020) 36:265-89. doi: 10.1146/ annurev-cellbio-021820-101354

26. De Leo MG, Staiano L, Vicinanza M, Luciani A, Carissimo A, Mutarelli M, et al. Autophagosome-Lysosome Fusion Triggers a Lysosomal Response Mediated by TLR9 and Controlled by OCRL. Nat Cell Biol (2016) 18(8):839_ 50. doi: $10.1038 /$ ncb3386

27. Oka T, Hikoso S, Yamaguchi O, Taneike M, Takeda T, Tamai T, et al Mitochondrial DNA That Escapes From Autophagy Causes Inflammation and Heart Failure. Nature (2012) 485(7397):251-5. doi: 10.1038/ nature 10992 
28. Riley JS, Tait SW. Mitochondrial DNA in Inflammation and Immunity. EMBO Rep (2020) 21(4):e49799. doi: 10.15252/embr.201949799

29. Zhong F, Liang S, Zhong Z. Emerging Role of Mitochondrial DNA as a Major Driver of Inflammation and Disease Progression. Trends Immunol (2019) 40(12):1120-33. doi: 10.1016/j.it.2019.10.008

30. Perez-Trevino P, Velasquez M, Garcia N. Mechanisms of Mitochondrial DNA Escape and its Relationship With Different Metabolic Diseases. Biochim Biophys Acta Mol Basis Dis (2020) 1866(6):165761. doi: 10.1016/ j.bbadis.2020.165761

31. Benmerzoug S, Ryffel B, Togbe D, Quesniaux VFJ. Self-DNA Sensing in Lung Inflammatory Diseases. Trends Immunol (2019) 40(8):719-34. doi: 10.1016/j.it.2019.06.001

32. White MJ, McArthur K, Metcalf D, Lane RM, Cambier JC, Herold MJ, et al. Apoptotic Caspases Suppress mtDNA-Induced STING-Mediated Type I IFN Production. Cell (2014) 159(7):1549-62. doi: 10.1016/j.cell.2014.11.036

33. Rongvaux A, Jackson R, Harman CC, Li T, West AP, de Zoete MR, et al. Apoptotic Caspases Prevent the Induction of Type I Interferons by Mitochondrial DNA. Cell (2014) 159(7):1563-77. doi: 10.1016/j.cell. 2014.11.037

34. Riley JS, Quarato G, Cloix C, Lopez J, O'Prey J, Pearson M, et al. Mitochondrial Inner Membrane Permeabilisation Enables mtDNA Release During Apoptosis. EMBO J (2018) 37(17):e99238. doi: 10.15252/embj. 201899238

35. McArthur K, Whitehead LW, Heddleston JM, Li L, Padman BS, Oorschot V, et al. BAK/BAX Macropores Facilitate Mitochondrial Herniation and mtDNA Efflux During Apoptosis. Science (2018) 359(6378):eaao6047. doi: 10.1126/science.aao6047

36. Bock FJ, Tait SWG. Mitochondria as Multifaceted Regulators of Cell Death. Nat Rev Mol Cell Biol (2020) 21(2):85-100. doi: 10.1038/s41580-019-0173-8

37. Carraro M, Bernardi P. Measurement of Membrane Permeability and the Mitochondrial Permeability Transition. Methods Cell Biol (2020) 155:36979. doi: 10.1016/bs.mcb.2019.10.004

38. Patrushev M, Kasymov V, Patrusheva V, Ushakova T, Gogvadze V, Gaziev A. Mitochondrial Permeability Transition Triggers the Release of mtDNA Fragments. Cell Mol Life Sci (2004) 61(24):3100-3. doi: 10.1007/s00018-0044424-1

39. Kanneganti TD, Kundu M, Green DR. Innate Immune Recognition of mtDNA-An Undercover Signal? Cell Metab (2015) 21(6):793-4. doi: 10.1016/j.cmet.2015.05.019

40. Kukat C, Wurm CA, Spahr H, Falkenberg M, Larsson NG, Jakobs S. Super-Resolution Microscopy Reveals That Mammalian Mitochondrial Nucleoids Have a Uniform Size and Frequently Contain a Single Copy of mtDNA. Proc Natl Acad Sci USA (2011) 108(33):13534-9. doi: 10.1073/ pnas. 1109263108

41. Sun B, Sundstrom KB, Chew JJ, Bist P, Gan ES, Tan HC, et al. Dengue Virus Activates cGAS Through the Release of Mitochondrial DNA. Sci Rep (2017) 7(1):3594. doi: 10.1038/s41598-017-03932-1

42. Zheng Y, Liu Q, Wu Y, Ma L, Zhang Z, Liu T, et al. Zika Virus Elicits Inflammation to Evade Antiviral Response by Cleaving cGAS via NS1Caspase-1 Axis. EMBO J (2018) 37(18):e99347. doi: 10.15252/embj. 201899347

43. Moriyama M, Koshiba T, Ichinohe T. Influenza A Virus M2 Protein Triggers Mitochondrial DNA-Mediated Antiviral Immune Responses. Nat Commun (2019) 10(1):4624. doi: 10.1038/s41467-019-12632-5

44. West AP, Khoury-Hanold W, Staron M, Tal MC, Pineda CM, Lang SM, et al. Mitochondrial DNA Stress Primes the Antiviral Innate Immune Response. Nature (2015) 520(7548):553-7. doi: 10.1038/nature14156

45. Tiku V, Tan MW, Dikic I. Mitochondrial Functions in Infection and Immunity: (Trends in Cell Biology 30, 263-275, 2020). Trends Cell Biol (2020) 30(9):748. doi: 10.1016/j.tcb.2020.07.001

46. Tigano M, Vargas DC, Tremblay-Belzile S, Fu Y, Sfeir A. Nuclear Sensing of Breaks in Mitochondrial DNA Enhances Immune Surveillance. Nature (2021) 591(7850):477-81. doi: 10.1038/s41586-021-03269-w

47. Wu Z, Sainz AG, Shadel GS. Mitochondrial DNA: Cellular Genotoxic Stress Sentinel. Trends Biochem Sci (2021) S0968-0004(21):00106-7. doi: 10.1016/ j.tibs.2021.05.004

48. Caielli S, Athale S, Domic B, Murat E, Chandra M, Banchereau R, et al. Oxidized Mitochondrial Nucleoids Released by Neutrophils Drive Type I
Interferon Production in Human Lupus. J Exp Med (2016) 213(5):697-713. doi: $10.1084 /$ jem. 20151876

49. Lood C, Blanco LP, Purmalek MM, Carmona-Rivera C, De Ravin SS, Smith CK, et al. Neutrophil Extracellular Traps Enriched in Oxidized Mitochondrial DNA are Interferogenic and Contribute to Lupus-Like Disease. Nat Med (2016) 22(2):146-53. doi: 10.1038/nm.4027

50. Doherty E, Oaks Z, Perl A. Increased Mitochondrial Electron Transport Chain Activity at Complex I is Regulated by N-Acetylcysteine in Lymphocytes of Patients With Systemic Lupus Erythematosus. Antioxid Redox Signal (2014) 21(1):56-65. doi: 10.1089/ars.2013.5702

51. Lai ZW, Hanczko R, Bonilla E, Caza TN, Clair B, Bartos A, et al. NAcetylcysteine Reduces Disease Activity by Blocking Mammalian Target of Rapamycin in T Cells From Systemic Lupus Erythematosus Patients: A Randomized, Double-Blind, Placebo-Controlled Trial. Arthritis Rheum (2012) 64(9):2937-46. doi: 10.1002/art.34502

52. Li S, Li H, Zhang YL, Xin QL, Guan ZQ, Chen X, et al. SFTSV Infection Induces BAK/BAX-Dependent Mitochondrial DNA Release to Trigger NLRP3 Inflammasome Activation. Cell Rep (2020) 30(13):4370-85.e7. doi: 10.1016/j.celrep.2020.02.105

53. Brokatzky D, Dorflinger B, Haimovici A, Weber A, Kirschnek S, Vier J, et al. A non-Death Function of the Mitochondrial Apoptosis Apparatus in Immunity. EMBO J (2019) 38(11):e100907. doi: 10.15252/embj.2018100907

54. Kerur N, Fukuda S, Banerjee D, Kim Y, Fu D, Apicella I, et al. cGAS Drives Noncanonical-Inflammasome Activation in Age-Related Macular Degeneration. Nat Med (2018) 24(1):50-61. doi: 10.1038/nm.4450

55. He WR, Cao LB, Yang YL, Hua D, Hu MM, Shu HB. VRK2 is Involved in the Innate Antiviral Response by Promoting Mitostress-Induced mtDNA Release. Cell Mol Immunol (2021) 18(5):1186-96. doi: 10.1038/s41423021-00673-0

56. Sprenger HG, MacVicar T, Bahat A, Fiedler KU, Hermans S, Ehrentraut D, et al. Cellular Pyrimidine Imbalance Triggers Mitochondrial DNADependent Innate Immunity. Nat Metab (2021) 3(5):636-650. doi: 10.1038/s42255-021-00385-9

57. Hu X, Peng X, Lu C, Zhang X, Gan L, Gao Y, et al. Type I IFN Expression is Stimulated by Cytosolic MtDNA Released From Pneumolysin-Damaged Mitochondria via the STING Signaling Pathway in Macrophages. FEBS $J$ (2019) 286(23):4754-68. doi: 10.1111/febs.15001

58. Huang LS, Hong Z, Wu W, Xiong S, Zhong M, Gao X, et al. mtDNA Activates cGAS Signaling and Suppresses the YAP-Mediated Endothelial Cell Proliferation Program to Promote Inflammatory Injury. Immunity (2020) 52(3):475-86.e5. doi: 10.1016/j.immuni.2020.02.002

59. Flores-Romero H, Garcia-Saez AJ. MAVS-Induced Mitochondrial Membrane Remodeling. FEBS J (2019) 286(8):1540-2. doi: 10.1111/ febs. 14822

60. Hwang MS, Boulanger J, Howe JD, Albecka A, Pasche M, Muresan L, et al. MAVS Polymers Smaller Than 80 Nm Induce Mitochondrial Membrane Remodeling and Interferon Signaling. FEBS J (2019) 286(8):1543-60. doi: 10.1111/febs. 14772

61. Wiatrek DM, Candela ME, Sedmik J, Oppelt J, Keegan LP, O'Connell MA. Activation of Innate Immunity by Mitochondrial dsRNA in Mouse Cells Lacking P53 Protein. RNA (New York NY) (2019) 25(6):713-26. doi: 10.1261/rna.069625.118

62. Vaseva AV, Marchenko ND, Ji K, Tsirka SE, Holzmann S, Moll UM. P53 Opens the Mitochondrial Permeability Transition Pore to Trigger Necrosis. Cell (2012) 149(7):1536-48. doi: 10.1016/j.cell.2012.05.014

63. Lee H, Fenster RJ, Pineda SS, Gibbs WS, Mohammadi S, Davila-Velderrain J, et al. Cell Type-Specific Transcriptomics Reveals That Mutant Huntingtin Leads to Mitochondrial RNA Release and Neuronal Innate Immune Activation. Neuron (2020) 107(5):891-908.e8. doi: 10.1016/j.neuron. 2020.06.021

64. Rai P, Janardhan KS, Meacham J, Madenspacher JH, Lin WC, Karmaus PWF, et al. IRGM1 Links Mitochondrial Quality Control to Autoimmunity. Nat Immunol (2021) 22(3):312-21. doi: 10.1038/s41590-020-00859-0

65. Pfanner N, Warscheid B, Wiedemann N. Mitochondrial Proteins: From Biogenesis to Functional Networks. Nat Rev Mol Cell Biol (2019) 20(5):26784. doi: 10.1038/s41580-018-0092-0

66. Kim Y, Park J, Kim S, Kim M, Kang MG, Kwak C, et al. PKR Senses Nuclear and Mitochondrial Signals by Interacting With Endogenous Double- 
Stranded RNAs. Mol Cell (2018) 71(6):1051-63.e6. doi: 10.1016/ j.molcel.2018.07.029

67. Li T, Huang T, Du M, Chen X, Du F, Ren J, et al. Phosphorylation and Chromatin Tethering Prevent cGAS Activation During Mitosis. Science (2021) 371(6535):eabc5386. doi: 10.1126/science.abc5386

68. Lazarou M. Keeping the Immune System in Check: A Role for Mitophagy. Immunol Cell Biol (2015) 93(1):3-10. doi: 10.1038/icb.2014.75

69. Lindqvist LM, Frank D, McArthur K, Dite TA, Lazarou M, Oakhill JS, et al. Autophagy Induced During Apoptosis Degrades Mitochondria and Inhibits Type I Interferon Secretion. Cell Death Differ (2018) 25(4):784-96. doi: 10.1038/s41418-017-0017-z

70. Yang B, Dan X, Hou Y, Lee JH, Wechter N, Krishnamurthy S, et al. NAD(+) Supplementation Prevents STING-Induced Senescence in Ataxia Telangiectasia by Improving Mitophagy. Aging Cell (2021) 20(4):e13329. doi: 10.1111/acel.13329

71. Zhang L, Qin Y, Chen M. Viral Strategies for Triggering and Manipulating Mitophagy. Autophagy (2018) 14(10):1665-73. doi: 10.1080/15548627. 2018.1466014

72. Vilmen G, Glon D, Siracusano G, Lussignol M, Shao Z, Hernandez E, et al. BHRF1, a BCL2 Viral Homolog, Disturbs Mitochondrial Dynamics and Stimulates Mitophagy to Dampen Type I IFN Induction. Autophagy (2021) 17(6):1295-315. doi: 10.1080/15548627.2020.1758416

73. Yang K, Huang R, Fujihira H, Suzuki T, Yan N. N-Glycanase NGLY1 Regulates Mitochondrial Homeostasis and Inflammation Through Nrf1. J Exp Med (2018) 215(10):2600-16. doi: 10.1084/jem.20180783

74. Xu Y, Shen J, Ran Z. Emerging Views of Mitophagy in Immunity and Autoimmune Diseases. Autophagy (2020) 16(1):3-17. doi: 10.1080/ 15548627.2019.1603547

75. Caza TN, Fernandez DR, Talaber G, Oaks Z, Haas M, Madaio MP, et al. HRES-1/Rab4-Mediated Depletion of Drp1 Impairs Mitochondrial Homeostasis and Represents a Target for Treatment in SLE. Ann Rheum Dis (2014) 73(10):1888-97. doi: 10.1136/annrheumdis-2013-203794

76. Dhir A, Dhir S, Borowski LS, Jimenez L, Teitell M, Rotig A, et al. Mitochondrial Double-Stranded RNA Triggers Antiviral Signalling in Humans. Nature (2018) 560(7717):238-42. doi: 10.1038/s41586-0180363-0

77. Lepelley A, Mina ED, Van Nieuwenhove E, Waumans L, Fraitag S, Rice GI, et al. Enhanced cGAS-STING-Dependent Interferon Signaling Associated With Mutations in ATAD3A. bioRxiv (2021) 218(10):e20201560. doi: 10.1084/jem.20201560

78. Pennisi A, Rotig A, Roux CJ, Levy R, Henneke M, Gartner J, et al. Heterogeneity of PNPT1 Neuroimaging: Mitochondriopathy, Interferonopathy or Both? J Med Genet (2020) jmedgenet-2020-107367. doi: 10.1136/jmedgenet-2020-107367

79. Lam C, Ferreira C, Krasnewich D, Toro C, Latham L, Zein WM, et al. Prospective Phenotyping of NGLY1-CDDG, the First Congenital Disorder of Deglycosylation. Genet Med (2017) 19(2):160-8. doi: 10.1038/gim.2016.75

80. Enns GM, Shashi V, Bainbridge M, Gambello MJ, Zahir FR, Bast T, et al. Mutations in NGLY1 Cause an Inherited Disorder of the Endoplasmic Reticulum-Associated Degradation Pathway. Genet Med (2014) 16(10):7518. doi: $10.1038 /$ gim.2014.22

81. Barrat FJ, Elkon KB, Fitzgerald KA. Importance of Nucleic Acid Recognition in Inflammation and Autoimmunity. Annu Rev Med (2016) 67:323-36. doi: 10.1146/annurev-med-052814-023338

82. Skaug B, Assassi S. Type I Interferon Dysregulation in Systemic Sclerosis. Cytokine (2020) 132:154635. doi: 10.1016/j.cyto.2018.12.018

83. Ryu C, Walia A, Ortiz V, Perry C, Woo S, Reeves BC, et al. Bioactive Plasma Mitochondrial DNA Is Associated With Disease Progression in Scleroderma-Associated Interstitial Lung Disease. Arthritis Rheumatol (2020) 72(11):1905-15. doi: 10.1002/art.41418

84. Harel T, Yoon WH, Garone C, Gu S, Coban-Akdemir Z, Eldomery MK, et al. Recurrent De Novo and Biallelic Variation of ATAD3A, Encoding a Mitochondrial Membrane Protein, Results in Distinct Neurological Syndromes. Am J Hum Genet (2016) 99(4):831-45. doi: 10.1016/ j.ajhg.2016.08.007

85. Cooper HM, Yang Y, Ylikallio E, Khairullin R, Woldegebriel R, Lin KL, et al. ATPase-Deficient Mitochondrial Inner Membrane Protein ATAD3A Disturbs Mitochondrial Dynamics in Dominant Hereditary Spastic
Paraplegia. Hum Mol Genet (2017) 26(8):1432-43. doi: 10.1093/hmg/ $\mathrm{ddx} 042$

86. Baudier J. ATAD3 Proteins: Brokers of a Mitochondria-Endoplasmic Reticulum Connection in Mammalian Cells. Biol Rev Camb Philos Soc (2018) 93(2):827-44. doi: 10.1111/brv.12373

87. Gilquin B, Taillebourg E, Cherradi N, Hubstenberger A, Gay O, Merle N, et al. The AAA+ ATPase ATAD3A Controls Mitochondrial Dynamics at the Interface of the Inner and Outer Membranes. Mol Cell Biol (2010) 30 (8):1984-96. doi: 10.1128/MCB.00007-10

88. Peralta S, Goffart S, Williams SL, Diaz F, Garcia S, Nissanka N, et al. ATAD3 Controls Mitochondrial Cristae Structure in Mouse Muscle, Influencing mtDNA Replication and Cholesterol Levels. J Cell Sci (2018) 131(13): jcs217075. doi: $10.1242 /$ jcs. 217075

89. Zhao Y, Sun X, Hu D, Prosdocimo DA, Hoppel C, Jain MK, et al. ATAD3A Oligomerization Causes Neurodegeneration by Coupling Mitochondrial Fragmentation and Bioenergetics Defects. Nat Commun (2019) 10 (1):1371. doi: 10.1038/s41467-019-09291-x

90. Lang L, Loveless R, Teng Y. Emerging Links Between Control of Mitochondrial Protein ATAD3A and Cancer. Int J Mol Sci (2020) 21 (21):7197. doi: 10.3390/ijms21217917

91. Fremond ML, Melki I, Kracker S, Bondet V, Duffy D, Rice GI, et al. Comment on: 'Aberrant tRNA Processing Causes an Autoinflammatory Syndrome Responsive to TNF Inhibitors' by Giannelou Et Al: Mutations in TRNT1 Result in a Constitutive Activation of Type I Interferon Signalling. Ann rheumatic Dis (2019) 78(8):e86. doi: 10.1136/annrheumdis-2018213745

92. Tonduti D, Orcesi S, Jenkinson EM, Dorboz I, Renaldo F, Panteghini C, et al. Clinical, Radiological and Possible Pathological Overlap of Cystic Leukoencephalopathy Without Megalencephaly and Aicardi-Goutieres Syndrome. Eur J Paediatr Neurol (2016) 20(4):604-10. doi: 10.1016/ j.ejpn.2016.03.009

93. Kameli R, Amanat M, Rezaei Z, Hosseionpour S, Nikbakht S, Alizadeh H, et al. RNASET2-Deficient Leukoencephalopathy Mimicking Congenital CMV Infection and Aicardi-Goutieres Syndrome: A Case Report With a Novel Pathogenic Variant. Orphanet J Rare Dis (2019) 14(1):184. doi: 10.1186/s13023-019-1155-9

94. Liu P, Huang J, Zheng Q, Xie L, Lu X, Jin J, et al. Mammalian Mitochondrial RNAs Are Degraded in the Mitochondrial Intermembrane Space by RNASET2. Protein Cell (2017) 8(10):735-49. doi: 10.1007/s13238-0170448-9

95. Sasarman F, Thiffault I, Weraarpachai W, Salomon S, Maftei C, Gauthier J, et al. The 3' Addition of CCA to Mitochondrial Trnaser(AGY) Is Specifically Impaired in Patients With Mutations in the tRNA Nucleotidyl Transferase Trnt1. Hum Mol Genet (2015) 24(10):2841-7. doi: 10.1093/hmg/ddv044

96. van der Burgh R, Nijhuis L, Pervolaraki K, Compeer EB, Jongeneel LH, van Gijn M, et al. Defects in Mitochondrial Clearance Predispose Human Monocytes to Interleukin-1beta Hypersecretion. J Biol Chem (2014) 289 (8):5000-12. doi: 10.1074/jbc.M113.536920

97. Mulders-Manders CM, Simon A. Hyper-IgD Syndrome/Mevalonate Kinase Deficiency: What Is New? Semin Immunopathol (2015) 37(4):371-6. doi: 10.1007/s00281-015-0492-6

98. Deshayes S, Georgin-Lavialle S, Hot A, Durel CA, Hachulla E, Rouanes N, et al. Efficacy of Continuous Interleukin 1 Blockade in Mevalonate Kinase Deficiency: A Multicenter Retrospective Study in 13 Adult Patients and Literature Review. J Rheumatol (2018) 45(3):425-9. doi: 10.3899/ jrheum. 170684

99. Bader-Meunier B, Florkin B, Sibilia J, Acquaviva C, Hachulla E, Grateau G, et al. Mevalonate Kinase Deficiency: A Survey of 50 Patients. Pediatrics (2011) 128(1):e152-9. doi: 10.1542/peds.2010-3639

100. Carapito R, Carapito C, Morlon A, Paul N, Vaca Jacome AS, Alsaleh G, et al. Multi-OMICS Analyses Unveil STAT1 as a Potential Modifier Gene in Mevalonate Kinase Deficiency. Ann Rheum Dis (2018) 77(11):1675-87. doi: 10.1136/annrheumdis-2018-213524

101. Georgel P. Crosstalk Between Interleukin-1beta and Type I Interferons Signaling in Autoinflammatory Diseases. Cells (2021) 10(5):1134. doi: $10.3390 /$ cells 10051134

102. Aarreberg LD, Esser-Nobis K, Driscoll C, Shuvarikov A, Roby JA, Gale MJr. Interleukin-1beta Induces mtDNA Release to Activate Innate Immune 
Signaling via cGAS-STING. Mol Cell (2019) 74(4):801-15.e6. doi: 10.1016/ j.molcel.2019.02.038

103. Wolf AR, Mootha VK. Functional Genomic Analysis of Human Mitochondrial RNA Processing. Cell Rep (2014) 7(3):918-31. doi: 10.1016/j.celrep.2014.03.035

104. Van Haute L, Pearce SF, Powell CA, D'Souza AR, Nicholls TJ, Minczuk M. Mitochondrial Transcript Maturation and its Disorders. J Inherit Metab Dis (2015) 38(4):655-80. doi: 10.1007/s10545-015-9859-z

105. Liu H, Golji J, Brodeur LK, Chung FS, Chen JT, deBeaumont RS, et al. Tumor-Derived IFN Triggers Chronic Pathway Agonism and Sensitivity to ADAR Loss. Nat Med (2019) 25(1):95-102. doi: 10.1038/s41591-018-0302-5

106. Stenton SL, Prokisch H. Genetics of Mitochondrial Diseases: Identifying Mutations to Help Diagnosis. EBioMedicine (2020) 56:102784. doi: 10.1016/ j.ebiom.2020.102784

107. Ng YS, Bindoff LA, Gorman GS, Klopstock T, Kornblum C, Mancuso M, et al. Mitochondrial Disease in Adults: Recent Advances and Future Promise. Lancet Neurol (2021) 20(7):573-84. doi: 10.1016/S1474-4422(21)00098-3

108. Suomalainen A, Battersby BJ. Mitochondrial Diseases: The Contribution of Organelle Stress Responses to Pathology. Nat Rev Mol Cell Biol (2018) 19 (2):77-92. doi: 10.1038/nrm.2017.66

109. Alston CL, Stenton SL, Hudson G, Prokisch H, Taylor RW. The Genetics of Mitochondrial Disease: Dissecting Mitochondrial Pathology Using MultiOmic Pipelines. J Pathol (2021) 254(4):430-42. doi: 10.1002/path.5641

110. Martinelli DC, Moneta I, Di Nottia GM, Piemonte M, Carrozzo F, Insalaco $\mathrm{R}$, et al. Interferon Signature: A New Biomarker to Follow Disease Progression in Pearson and Kearns-Sayre Syndrome (Abstract). J Inherit Metab Dis (2019) 42(Suppl 1):1-479.

111. Torres-Odio S, Lei Y, Gispert S, Maletzko A, Key J, Menissy SS, et al. Loss of Mitochondrial Protease CLPP Activates Type I IFN Responses Through the Mitochondrial DNA-cGAS-STING Signaling Axis. J Immunol (2021) 206 (8):1890-900. doi: 10.4049/jimmunol.2001016

112. Lei Y, Martinez CG, Torres-Odio S, Bell SL, Birdwell CE, Bryant JD, et al. Type I Interferon Potentiates Metabolic Dysfunction, Inflammation, and Accelerated Aging in mtDNA Mutator Mice. bioRxiv (2020) 7(22):eabe7548. doi: 10.1126/sciadv.abe7548

113. Kim H, de Jesus AA, Brooks SR, Liu Y, Huang Y, VanTries R, et al. Development of a Validated Interferon Score Using NanoString Technology. J Interferon Cytokine Res Off J Int Soc Interferon Cytokine Res (2018) 38(4):171-85. doi: 10.1089/jir.2017.0127

114. Rice GI, Melki I, Fremond ML, Briggs TA, Rodero MP, Kitabayashi N, et al. Assessment of Type I Interferon Signaling in Pediatric Inflammatory Disease. J Clin Immunol (2017) 37(2):123-32. doi: 10.1007/s10875-016-0359-1

115. Feichtinger RG, Sperl W, Bauer JW, Kofler B. Mitochondrial Dysfunction: A Neglected Component of Skin Diseases. Exp Dermatol (2014) 23(9):607-14. doi: $10.1111 /$ exd.12484

116. Finsterer J, Melichart-Kotig M, Woehrer A. Mitochondrial Disorder Mimicking Rheumatoid Disease. Z Rheumatol (2019) 78(9):875-80. doi: 10.1007/s00393-018-0551-1

117. Walker MA, Slate N, Alejos A, Volpi S, Iyengar RS, Sweetser D, et al. Predisposition to Infection and SIRS in Mitochondrial Disorders: 8 Years' Experience in an Academic Center. J Allergy Clin Immunol Pract (2014) 2 (4):465-8.el. doi: 10.1016/j.jaip.2014.02.009

118. Finsterer J, Zarrouk-Mahjoub S. Cerebral Imaging in Paediatric Mitochondrial Disorders. Neuroradiol J (2018) 31(6):596-608. doi: $10.1177 / 1971400918786054$

119. Livingston JH, Lin JP, Dale RC, Gill D, Brogan P, Munnich A, et al. A Type I Interferon Signature Identifies Bilateral Striatal Necrosis Due to Mutations in
ADAR1. J Med Genet (2014) 51(2):76-82. doi: 10.1136/jmedgenet-2013102038

120. Crow YJ, Chase DS, Lowenstein Schmidt J, Szynkiewicz M, Forte GM, Gornall HL, et al. Characterization of Human Disease Phenotypes Associated With Mutations in TREX1, RNASEH2A, RNASEH2B, RNASEH2C, SAMHD1, ADAR, and IFIH1. Am J Med Genet Part A (2015) 167A(2):296-312. doi: 10.1002/ajmg.a.36887

121. Crow YJ, Zaki MS, Abdel-Hamid MS, Abdel-Salam G, Boespflug-Tanguy O, Cordeiro NJ, et al. Mutations in ADAR1, IFIH1, and RNASEH2B Presenting as Spastic Paraplegia. Neuropediatrics (2014) 45(6):386-93. doi: 10.1055/s0034-1389161

122. Vanderver A, Adang L, Gavazzi F, McDonald K, Helman G, Frank DB, et al. Janus Kinase Inhibition in the Aicardi-Goutieres Syndrome. $N$ Engl J Med (2020) 383(10):986-9. doi: 10.1056/NEJMc2001362

123. Rodero MP, Fremond ML, Rice GI, Neven B, Crow YJ. JAK Inhibition in STING-Associated Interferonopathy. Ann Rheum Dis (2016) 75(12):e75. doi: 10.1136/annrheumdis-2016-210504

124. Jiang J, Zhao $\mathrm{M}$, Chang $\mathrm{C}, \mathrm{Wu} \mathrm{H}, \mathrm{Lu}$ Q. Type I Interferons in the Pathogenesis and Treatment of Autoimmune Diseases. Clin Rev Allergy Immunol (2020) 59(2):248-72. doi: 10.1007/s12016-020-08798-2

125. Haag SM, Gulen MF, Reymond L, Gibelin A, Abrami L, Decout A, et al. Targeting STING With Covalent Small-Molecule Inhibitors. Nature (2018) 559(7713):269-73. doi: 10.1038/s41586-018-0287-8

126. Russell OM, Gorman GS, Lightowlers RN, Turnbull DM. Mitochondrial Diseases: Hope for the Future. Cell (2020) 181(1):168-88. doi: 10.1016/ j.cell.2020.02.051

127. West AP. Mitochondrial Dysfunction as a Trigger of Innate Immune Responses and Inflammation. Toxicology (2017) 391:54-63. doi: 10.1016/ j.tox.2017.07.016

128. Montava-Garriga L, Ganley IG. Outstanding Questions in Mitophagy: What We Do and Do Not Know. J Mol Biol (2020) 432(1):206-30. doi: 10.1016/ j.jmb.2019.06.032

129. Cooper MA, Zimmerman O, Nataraj R, Wynn RF. Lifelong Immune Modulation Versus Hematopoietic Cell Therapy for Inborn Errors of Immunity. J Allergy Clin Immunol Pract (2021) 9(2):628-39. doi: 10.1016/ j.jaip.2020.11.055

130. Johnson SC, Martinez F, Bitto A, Gonzalez B, Tazaerslan C, Cohen C, et al. mTOR Inhibitors may Benefit Kidney Transplant Recipients With Mitochondrial Diseases. Kidney Int (2019) 95(2):455-66. doi: 10.1016/ j.kint.2018.08.038

Conflict of Interest: The authors declare that the research was conducted in the absence of any commercial or financial relationships that could be construed as a potential conflict of interest.

Publisher's Note: All claims expressed in this article are solely those of the authors and do not necessarily represent those of their affiliated organizations, or those of the publisher, the editors and the reviewers. Any product that may be evaluated in this article, or claim that may be made by its manufacturer, is not guaranteed or endorsed by the publisher.

Copyright $\odot 2021$ Lepelley, Wai and Crow. This is an open-access article distributed under the terms of the Creative Commons Attribution License (CC BY). The use, distribution or reproduction in other forums is permitted, provided the original author(s) and the copyright owner(s) are credited and that the original publication in this journal is cited, in accordance with accepted academic practice. No use, distribution or reproduction is permitted which does not comply with these terms. 